\title{
ON FUNCTIONS OF BOUNDED VARIATION RELATIVE TO A SET
}

\author{
P. C. BHAKTA \\ (Received 24 December 1968; revised 10 March 1970) \\ Communicated by E. Strzelecki
}

\section{Introduction, Definitions and Notations}

The present paper on functions of bounded variation relative to a set has its point of departure in the work of R. L. Jeffery [10]. Below we recapitulate Jeffery's class $U$ of functions of bounded variation relative to a set, we state and prove a number of preliminary lemmas and theorems, we introduce a suitable pseudometric space $(X, d)$ of such functions, and the analogous space $(\bar{X}, \rho)$, and prove that $(X, d)$ is separable, that every closed sphere in $(X, d)$ is compact and that $(\bar{X}, \rho)$ is complete. These results extend known results of C. R. Adams, and C. R. Adams and A. P. Morse for the space of usual $B V$ functions.

Let $S$ be a subset of the closed interval $[a, b]$ such that $S$ is dense in $[a, b]$. We define the class $U$ of functions $F(x)$ in the following way: $F(x)$ is defined on $[a, b]$ such that for every point $x_{0}$ in $[a, b], F(x)$ tends to finite limits as $x$ tends to $x_{0}+$ and to $x_{0}-$ over the points of $S$; these limits will be denoted by $F\left(x_{0}+\right)$ and $F\left(x_{0}-\right)$ respectively.

We now introduce the following definition:

Definition 1.1. Let $F(x)$ belong to the class $U$ and $E$ be a subset of $[a, b]$ with $\alpha$ and $\beta$ as its g.l.b. and l.u.b. Let

$$
D:\left(\alpha<x_{1}<x_{2}<\cdots<x_{p}<\beta\right)
$$

be any subdivision of $[\alpha, \beta]$ with $x_{i} \in E$. The l.u.b. of the sums $V_{D}$ defined by

$$
V_{D}=\left|F(x)-F\left(x_{1}-\right)\right|+\sum_{i=1}^{r-1}\left|F\left(x_{i}+\right)-F\left(x_{i+1}-\right)\right|+\left|F\left(x_{r}+\right)-F(\beta)\right|
$$

for all possible subdivisions $D$ is called the total variation, $V_{S}(F ; E)$, of $F(x)$ on $E$ relative to the set $S$. If $V_{S}(F ; E)<+\infty$, then $F(x)$ is said to be $B V-S$ on $E$.

From theorem 3.1 and lemma 2.2 it follows that a function which is of bounded variation in the ordinary sense on $[a, b]$ is a $B V-S$ function and the $B V-S$ variation of a function $F(x)$ on a dense subset of $[a, b]$ is the same as that of the ordinary variation of the function $F(x-)$ [or $F(x+)]$ on $[a, b]$. However, 
there are examples of functions that are $B V-S$ on an infinite subset $E$ of $[a, b]$ but not $B V$ on $E$. Therefore the set of functions which are $B V-S$ on $[a, b]$ that is, the set of functions $F(x)$ for which the total variation of $F(x-)$ [or $F(x+)$ ] on $[a, b]$ is finite $\}$ includes as a proper subset the functions which are $B V$ on $[a, b]$. It may be noted in this connection that various authors have studied the properties of $B V$ functions on a set. These studies can be found in most of the references appended in the list of the bibliography.

Throughout our discussion we suppose that $S$ is a fixed set which is dense in $[a, b]$, and consequently $U$ becomes a fixed class of functions as defined above. We denote the set of points $x$ of $S$ for which $F(x-)=F(x)=F(x+)$ by $S_{F}$, where $S$ is as above and $F(x)$ is any function belonging to the class $U$. From theorem 3.4 onwards we suppose that $S$ is Lebesgue measurable and $m S=b-a$.

\section{Preliminary lemmas}

LEMMA 2.1. Let $F(x)$ belong to the class $U$. Then the set of points for which $F(x-) \neq F(x+)$ is countable. Also the subset of $S$ for which we do not have $F(x-)=F(x)=F(x+)$ is countable.

Proof. For each positive integer $n$, let $E_{n}$ denote the set of numbers $x$ such that

$$
|F(x-)-F(x+)|>\frac{1}{n}, \quad a+\frac{1}{n}<x<b-\frac{1}{n} .
$$

The set $E_{n}$ cannot have a cluster point, and hence it is finite. The set $\bigcup_{n=1}^{\infty} E_{n}$ is therefore countable. Similarly for the second part of the lemma and this completes the proof.

We now define the function $G(x)$ on $[a, b]$ as follows:

$$
\begin{aligned}
& G(a)=F(a), \quad G(b)=F(b), \\
& G(x)=F(x-) \text { for } a<x<b .
\end{aligned}
$$

It is easy to verify that

$$
F(x+)=\lim G(\eta) \text { as }(\eta>x, \eta \rightarrow x),
$$

and

$$
G(x)=\lim F(\xi+) \text { as }(\xi<x, \xi \rightarrow x) .
$$

Clearly $G(x)=F(x)$ at each point of the set $S_{F}$.

LEMMA 2.2. If $E$ is dense in $[a, b]$ and if $F(x)$ belongs to $U$, then

$$
V_{S}(F ; E)=V_{a}^{b}(G) \text {. }
$$

Proof. The symbol $\approx$ shall mean: can be made to differ by $\varepsilon(>0)$ by going far enough in the limiting process indicated by $\rightarrow$. The symbols $x_{0}+$ and $x_{r+1}-$ shall mean $a$ and $b$ respectively. 
To prove $V_{S}(F ; E) \leqq V_{a}^{b}(G)$ we consider any subdivision

$$
D:\left(a=x_{0}<x_{1}<x_{2}<\cdots<x_{r+1}=b\right)
$$

of $[a, b]$ with $x_{i} \in E(i=1,2, \cdots, r)$ and then take points $\eta_{i}$ satisfying $x_{i}<\eta_{i}<x_{i+1}$ and $\eta_{i} \rightarrow x_{i}$ (except for $\left.\eta_{0}=a, \eta_{r+1}=b\right)$. Denoting by $V_{D}$ the sum

we have

$$
\sum_{i=0}^{r}\left|F\left(x_{i}+\right)-F\left(x_{i+1}-\right)\right|,
$$

$$
V_{D} \approx \sum_{i=0}^{r}\left|F\left(\eta_{i}-\right)-F\left(x_{i+1}-\right)\right|=\sum_{i=0}^{r}\left|G\left(\eta_{i}\right)-G\left(x_{i+1}\right)\right| \leqq V_{a}^{b}(G) .
$$

Hence

$$
V_{S}(F ; E) \leqq V_{a}^{b}(G)
$$

To prove the reverse inequality we consider any subdivision $D$ of $[a, b]$, then take points $\xi_{i}$ satisfying $\xi_{i} \in E, x_{i-1}<\xi_{i}<x_{i}$ (except for $\xi_{0}=a, \xi_{r+1}=b$ ). Then

$$
\sum_{i=0}^{r}\left|G\left(x_{i}\right)-G\left(x_{i+1}\right)\right| \approx \sum_{i=0}^{r}\left|F\left(\xi_{i}+\right)-F\left(\xi_{i+1}-\right)\right| \leqq V_{S}(F ; E) ;
$$

and hence

$$
V_{S}(F ; E)=V_{a}^{b}(G) .
$$

Corollary 2.2.1. If $E$ is dense in $[a, b]$, then $V_{S}(F ; E)=V_{S}(F ;[a, b])$.

LEMMA 2.3. Let $a<c<b$. If $F(x)$ is $B V-S$ on $[a, c]$ and on $[c, b]$, then it is so on $[a, b]$; further if $c \in S_{F}$ then

$$
V_{S}(F ;[a, b])=V_{S}(F ;[a, c])+V_{S}(F ;[c, b]) .
$$

LEMMA 2.4. If $F(x)$ is $B V-S$ on $[a, b]$, then $F(x+)$ is bounded on $[a, b]$. The proofs of these results are straightforward. lows:

Let $F(x)$ be $B V-S$ on $[a, b]$. We define the function $\pi(x)$ on $[a, b]$ as fol-

$$
\pi(a)=0 \text { and } \pi(x)=V_{a}^{x}(G) \text { for } a<x \leqq b .
$$

Clearly the function $\pi(x)$ is non-decreasing on $[a, b]$.

LEMMA 2.5. If $F(x)$ is $B V-S$ on $[a, b]$, then $F(x)$ can be expressed as $F(x)=\pi(x)-v(x)$, where $v(x)$ is non-decreasing on $S_{F}$.

Proof. We define $v(x)$ by $v(x)=\pi(x)-F(x)$. Let $x_{1}$ and $x_{2}\left(>x_{1}\right)$ be any two points of $S_{F}$. Then 


$$
\begin{aligned}
v\left(x_{2}\right)-v\left(x_{1}\right) & =\left\{\pi\left(x_{2}\right)-F\left(x_{2}\right)\right\}-\left\{\pi\left(x_{1}\right)-F\left(x_{1}\right)\right\} \\
& =\pi\left(x_{2}\right)-\pi\left(x_{1}\right)-\left\{G\left(x_{2}\right)-G\left(x_{1}\right)\right\} \\
& \geqq 0
\end{aligned}
$$

and the lemma is proved.

\section{Some results on $B V-S$ functions}

THEOREM 3.1. If $F(x)$ is of bounded variation on $[a, b]$, then it is $B V-S$ on $[a, b]$ and in any case

$$
V_{S}(F ;[a, b]) \leqq V_{a}^{b}(F), \quad F(x) \in U .
$$

Proof. We first suppose that $V_{a}^{b}(F)$ is finite. Let

$$
D:\left(a=x_{0}<x_{1}<x_{2}<\cdots<x_{r+1}=b\right)
$$

be any subdivision of $[a, b]$. Take points $\xi_{i}, \eta_{i}$ of $S$ with $x_{i}<\xi_{i}<\eta_{i}<x_{i+1}$ (except for $\xi_{0}=a, \eta_{r+1}=b$ ). Then

$$
\sum_{i=0}^{r}\left|F\left(\xi_{i}\right)-F\left(\eta_{i}\right)\right| \leqq V_{a}^{b}(F)
$$

Now letting $\xi_{i} \rightarrow x_{i}, \eta_{i} \rightarrow x_{i+1}$ over the points of $S$ we obtain

$$
\left|F(a)-F\left(x_{1}-\right)\right|+\sum_{i=1}^{r-1}\left|F\left(x_{i}+\right)-F\left(x_{i+1}-\right)\right|+\left|F\left(x_{r}+\right)-F(b)\right| \leqq V_{a}^{b}(F) .
$$

Since $D$ is arbitrary, we have

$$
V_{S}(F ;[a, b]) \leqq V_{a}^{b}(F) .
$$

So $F(x)$ is $B V-S$ on $[a, b]$. If $V_{a}^{b}(F)$ is infinite, then clearly (1) holds.

Note. It is clear that if $F(x)$ is monotone or continuous on $[a, b]$ then

$$
V_{S}(F ;[a, b])=V_{a}^{b}(F) .
$$

If $(B V)$ denotes the set of all functions which are of bounded variation on $[a, b]$ and $(B V-S)$ the set of all functions which are $B V-S$, then by Theorem $3.1,(B V) \subset(B V-S)$. The following example shows that $(B V)$ is a proper subset of $(B V-S)$.

ExAmple. Let $S$ be a dense subset of $[a, b]$ and let $E$ be an infinite subset of $[a, b]$ with $a$ and $b$ as lower and upper bounds. Let $\phi(x)$ be any non-decreasing function on $[a, b]$ and $\sum \beta_{n}$ be a divergent series of positive terms with $\lim \beta_{n}=0$. Choose a strictly monotone sequence $\left\{\alpha_{n}\right\}$ from $E$. Suppose that $\left\{\alpha_{n}\right\}$ is increasing. We define the function $F(x)$ on $[a, b]$ as follows: 


$$
\begin{aligned}
F(x) & =\phi(x)+\beta_{n} \text { for } x=\alpha_{2 n}(n=1,2,3, \cdots), \\
& =\phi(x) \text { elsewhere. }
\end{aligned}
$$

It is easy to see that $F(x \pm)=\phi(x \pm)$ for all $x \in[a, b]$. For any two points $c$, $d(>c)$ of $E$

$$
|F(c+)-F(d-)|=|\phi(c+)-\phi(d-)| \leqq \phi(d)-\phi(c)
$$

which shows that $F(x)$ is $B V-S$ on $E$. Now consider the subdivision $a \leqq \alpha_{1}<\alpha_{2}<\cdots<\alpha_{2 m}<b$ and denote by $V$ the sum

$$
\left|F(a)-F\left(\alpha_{1}\right) 1+\sum_{i=1}^{2 m-1}\right| F\left(\alpha_{i}\right)-F\left(\alpha_{i+1}\right)|+| F\left(\alpha_{2 m}\right)-F(b) \mid .
$$

Then

$$
\begin{aligned}
V & \geqq \sum_{i=1}^{m}\left|F\left(\alpha_{2 i-1}\right)-F\left(\alpha_{2 i}\right)\right| \\
& =\sum_{i=1}^{m}\left\{\phi\left(\alpha_{2 i}\right)+\beta_{i}-\phi\left(\alpha_{2 i-1}\right)\right\} \\
& \geqq \beta_{1}+\beta_{2}+\cdots+\beta_{m} .
\end{aligned}
$$

Since $\sum \beta_{n}$ is divergent, it follows that $F(x)$ is not of bounded variation on $E$.

THEOREM 3.2. (cf. [2], Th. 2; [4], Lemma 1; [9], § 7).

Let $\left\{F_{n}(x)\right\}$ be a sequence of functions in the class $U$ and $S_{0}=\cap\left\{S_{F_{n}}\right.$; $n=1,2, \cdots\}$. If $F_{n}(x) \rightarrow F(x) \in U$ at each point of the set $E \cup\{a, b\}$ such that $E \subset S_{0} \cap S_{F}$ and $E$ is dense in $[a, b]$, then

$$
\lim _{n \rightarrow \infty} \inf V_{S}\left(F_{n} ;[a, b]\right) \geqq V_{S}(F ;[a, b]) .
$$

Proof. We suppose that $V_{S}(F ; E)$ is finite. If $V_{S}(F ; E)$ is infinite the proof is analogous. Let $\varepsilon>0$ be arbitrary. There exists a subdivision

$$
D:\left(a=x_{0}<x_{1}<x_{2}<\cdots<x_{r+1}=b\right)
$$

with $x_{i} \in E(i=1,2, \cdots, r)$ such that

$$
\left|F(a)-F\left(x_{1}-\right)\right|+\sum_{i=1}^{r-1}\left|F\left(x_{i}+\right)-F\left(x_{i+1}-\right)\right|+\left|F\left(x_{r}+\right)-F(b)\right|>V_{S}(F ; E)-\varepsilon
$$

or

$$
V_{D}(F)=\sum_{i=0}^{r}\left|F\left(x_{i}\right)-F\left(x_{i+1}\right)\right|>V_{S}(F ; E)-\varepsilon .
$$

Since $V_{D}\left(F_{n}\right) \rightarrow V_{D}(F)$ as $n \rightarrow \infty$; a positive integer $n_{0}$ exists such that for $n \geqq n_{0}$

So,

$$
V_{S}\left(F_{n} ;[a, b]\right) \geqq V_{D}\left(F_{n}\right)>V_{S}(F ; E)-\varepsilon .
$$

$$
\lim _{n \rightarrow \infty} \inf V_{S}\left(F_{n} ;[a, b]\right) \geqq V_{S}(F ; E)-\varepsilon .
$$

Since $\varepsilon>0$ is arbitrary, we obtain by using corollary 2.2 .1 , 


$$
\lim _{n \rightarrow \infty} \inf V_{S}\left(F_{n} ;[a, b]\right) \geqq V_{S}(F ;[a, b]) .
$$

Definition 3.1. Let $F(x) \in U$ and $D:\left(a=x_{0}<x_{1}<x_{2}<\cdots<x_{r+1}=b\right)$ be any subdivision of $[a, b]$ with $x_{i} \in S_{F}(i=1,2, \cdots, r)$. We denote by $B(x)=$ $B(x ; F, D)$ the function whose graph is the polygonal line joining the points $\left(x_{i}, F\left(x_{i}\right)\right)(i=0,1,2, \cdots, r) . B(x)$ is said to be a Polygonal function associated with $F(x)$.

It is clear that

$$
V_{D}(F)=\sum_{i=0}^{r}\left|F\left(x_{i}\right)-F\left(x_{i+1}\right)\right|=V_{D}(B)=V_{S}(B ;[a, b]) .
$$

So,

$$
V_{S}(F ;[a, b]) \geqq V_{S}(B ;[a, b]) .
$$

THEOREM 3.3. (cf. [3], § 2). If $F(x)$ is $B V-S$ on $[a, b]$ and $S_{F}$ is dense in $[a, b]$, then it is possible to choose a sequence $\left\{B_{n}(x)\right\}$ of polygonal functions such that $B_{n}(x) \rightarrow F(x)$ at each point of $S_{F}$ and

$$
\lim _{n \rightarrow \infty} V_{S}\left(B_{n} ;[a, b]\right)=V_{S}(F ;[a, b]) .
$$

Proof. Let $\left\{D_{n}\right\}$ be a sequence of subdivisions

$$
D_{n}:\left(a=x_{0}^{(n)}<x_{1}^{(n)}<x_{2}^{(n)}<\cdots<x_{r_{n}+1}^{(n)}=b\right)
$$

of $[a, b]$ with $x_{i}^{(n)} \in S_{F}\left(i=1,2, \cdots, r_{n}\right)$ such that $D_{n} \subset D_{n+1}$ for each $n$ and the set $E=\bigcup\left\{D_{n} ; n=1,2, \cdots\right\}$ is dense in $[a, b]$. Writing $B_{n}(x)=B\left(x ; F, D_{n}\right)$ we have

$$
V_{S}\left(B_{n} ;[a, b]\right) \leqq V_{S}(F ;[a, b]) .
$$

Let $\varepsilon>0$ be arbitrary. Then a subdivision $D:\left(a=x_{0}<x_{1}<\cdots<x_{r+1}=b\right)$ with $x_{i} \in E(i=1,2, \cdots, r)$ exists such that

$$
\sum_{i=0}^{r}\left|F\left(x_{i}\right)-F\left(x_{i+1}\right)\right|>V_{S}(F ; E)-\varepsilon .
$$

Since $x_{i}$ 's are points of $E$, we can choose a positive integer $n_{0}$ such that $D \subset D_{n}$ for all $n \geqq n_{0}$.

Then for $n \geqq n_{0}$

$$
V_{S}\left(B_{n} ;[a, b]\right) \geqq \sum_{i=0}^{r} F\left(x_{i}\right)-F\left(x_{i+1}\right) \mid>V_{S}(F ; E)-\varepsilon .
$$

Combining (2) and (3) and noting corollary 2.2.1, we obtain

$$
\lim _{n \rightarrow \infty} V_{S}\left(B_{n} ;[a, b]\right)=V_{s}(F ;[a, b]) .
$$

It is clear that $B_{n}(x) \rightarrow F(x)$ at each point of the set $E$. Let $\xi$ be any point of $S_{F}-E$. Choose points $\xi^{\prime}, \xi^{\prime \prime}$ of $E$ with $\xi^{\prime}<\xi<\xi^{\prime \prime}$ such that $\pi\left(\xi^{\prime \prime}\right)-\pi\left(\xi^{\prime}\right)<\frac{1}{2} \varepsilon$. 
Let $m$ be a positive integer such that $\xi^{\prime}, \xi^{\prime \prime} \in D_{n}$ for all $n \geqq m$. Then for all $n \geqq m$

$$
\begin{aligned}
\left|F(\xi)-B_{n}(\xi)\right| & \leqq\left|F(\xi)-F\left(\xi^{\prime}\right)\right|+\left|F\left(\xi^{\prime}\right)-B_{n}\left(\xi^{\prime}\right)\right|+\left|B_{n}\left(\xi^{\prime}\right)-B_{n}(\xi)\right| \\
& \leqq\left|G(\xi)-G\left(\xi^{\prime}\right)\right|+V_{S}\left(F ;\left[\xi^{\prime}, \xi^{\prime \prime}\right]\right) \\
& \leqq 2\left[\pi\left(\xi^{\prime \prime}\right)-\pi\left(\xi^{\prime}\right)\right]<\varepsilon
\end{aligned}
$$

This proves the theorem.

THEOREM 3.4. (cf. [13], p. 222). Let $\mathscr{F}=\{F(x)\}$ be a sequence of functions in the class $U$. If there is a positive $K$ such that $|F(x \pm)| \leqq K, a<x<b ;|F(a)|$, $|F(b)| \leqq K$ and $V_{S}(F ;[a, b]) \leqq K$ for every $F(x) \in \mathscr{F}$, then there exist a subsequence in $\mathscr{F}$ which converges to a function $\phi(x)$ almost everywhere in $[a, b]$, where $\phi(x)$ is of bounded variation in $[a, b]$.

To prove the theorem we require the following lemma:

Lemma 3.1. (cf. [13], p. 221). Let $\mathscr{F}=\{F(x)\}$ be a sequence of functions in the class $U$ and $S_{0}=\cap\left\{S_{F} ; F \in \mathscr{F}\right\}$. If each $F(x)$ is non-decreasing on $S_{0}$ and if there is a positive $K$ such that $|F(x \pm)| \leqq K, a<x<b ;|F(a)|,|F(b)| \leqq K$ for each $F \in \mathscr{F}$, then there is a subsequence $\left\{F_{n}(x)\right\}$ of functions in $\mathscr{F}$ which converges to a function $\phi(x)$ almost everywhere in $[a, b]$, where $\phi(x)$ is non-decreasing on $[a, b]$.

The lemma can be proved in the usual way.

Proof of Theorem 3.4. By lemma 2.5, each $F(x)$ in $\mathscr{F}$ can be expressed as $F(x)=\pi(x)-v(x)$, where $\pi(x)$ is non-decreasing on $[a, b]$ and $v(x)$ is nondecreasing on $S_{0}=\cap\left\{S_{F} ; F \in \mathscr{F}\right\}$. Clearly $\pi(x)$ belongs to the class $U$. Also $V_{S}(\pi ;[a, b])=\pi(b)$. So $\pi(x) \leqq k$ for all $x \in[a, b]$. Since $v(x)=\pi(x)-F(x), v(x)$ belongs to the class $U$ and $|v(x \pm)| \leqq 2 k, a<x<b ;|v(a)|,|v(b)| \leqq 2 k$. By lemma 2 ([13], p. 221) there is a subsequence $\left\{\pi_{n}(x)\right\}$ of $\{\pi(x)\}$ which converges to a non-decreasing function $\alpha(x)$ everywhere in $[a, b]$.

Let $E_{n}$ denote the set of points in $[a, b]$, where

$$
v_{n}(x-)=v_{n}(x)=v_{n}(x+) \text { and } E_{0}=\cap\left\{E_{n} ; \quad n=1,2, \cdots\right\} .
$$

Then Lebesgue measure of $E_{0}$ is $b-a$. Applying lemma 3.1 to the sequence $\left\{v_{n}(x)\right\}$ (where $S_{0}$ is to be replaced by $S_{0} \cap E_{0}$ ) we obtain a subsequence $\left\{v_{n_{i}}(x)\right\}$ which converges to a non-decreasing function $\beta(x)$ almost everywhere in $[a, b]$. Let $\phi(x)=\alpha(x)-\beta(x)$. Then $\phi(x)$ is of bounded variation on $[a, b]$ and the sequence $\left\{F_{n_{i}}(x)\right\}$ converges to $\phi(x)$ almost everywhere in $[a, b]$. This proves the theorem.

\section{The space $(X, d)$}

Let $X$ denote the set of all functions $x(t)$ in the class $U$ which are $B V-S$ on $[0,1]$. To each pair $x, y$ of functions in $X$ we associate the real number $d(x, y)$ 
defined by

$$
d(x, y)=\int_{0}^{1}|x(t)-y(t)| d t+|T(x)-T(y)|,
$$

where the integral is taken in Lebesgue sense and $T(x)$ stands for $V_{S}(x ;[0,1])$. Since $d(x, y)=0$ implies $T(x)=T(y)$ and $x(t)=y(t)$ almost everywhere in $[0,1]$, it follows that $d$ is a pseudo-metric for $X$ and therefore $(X, d)$ is a pseudometric space.

The pseudo-metric (4) is analogous to that introduced by C. R. Adams [1] and C. R. Adams \& A. P. Morse [4] to study the properties of the space $(B V)$ of functions of bounded variation. The above two papers contain interesting and elaborate discussions of the space $(B V)$. Here we wish to mention only two properties of the space $(X, d)$ leaving out, of course, possible scope of further study.

THEOREM 4.1. The space $(X, d)$ is separable.

Proof. Let $E$ denote the set of all polygonal functions in $X$ with rational corners. Then clearly $E$ is countable. Let $x(t)$ be any function in $X$. By theorem 3.3 , it is possible to choose a sequence of polygonal functions $\left\{B_{n}(t)\right\}$ in $X$ such that $B_{n}(t) \rightarrow x(t)$ almost everywhere in $[0,1]$ and $T\left(B_{n}\right) \rightarrow T(x)$. For each $B_{n}(t)$ we can choose a polygonal function $P_{n}(t)$ in $X$ with rational comers such that $\left|B_{n}(t)-P_{n}(t)\right|<1 / n$ for all $t \in[0,1]$ and $\left|T\left(B_{n}\right)-T\left(P_{n}\right)\right|<1 / n$. So the sequence $\left\{P_{n}(t)\right\}$ converges to $x(t)$ almost everywhere in $[0,1]$ and $T\left(P_{n}\right) \rightarrow T(x)$. Therefore $d\left(P_{n}, x\right) \rightarrow 0$ as $n \rightarrow \rightarrow$ and hence $x$ is an accumulation point of $E$. Thus the set $E$ is dense in $X$. This completes the proof.

THEOREM 4.2. Every closed sphere in $(X, d)$ is compact.

Proof. Let $x_{0}$ be an element of $X$ and

$$
Y=\left\{x ; x \in X \text { and } d\left(x, x_{0}\right) \leqq r\right\}
$$

where $r$ is a positive number. Since $X$ is separable, $Y$ considered as a subspace of $X$ is a Lindelöf space. Let $\theta(t) \equiv 0$ in $[0,1]$. Then $\theta$ is an element of $X$. For any $x$ in $Y$,

So

$$
d(x, \theta) \leqq d\left(x, x_{0}\right)+d\left(x_{0}, \theta\right) \leqq r+d\left(x_{0}, \theta\right)
$$

where $M$ denotes the constant $r+d\left(x_{0}, \theta\right)$. If $t$ is a point in $(0,1)$, then

$$
|x(t \pm)| \leqq \max \{|x(0)|,|x(1)|\}+T(x) .
$$

We show that $\max \{|x(0)|,|x(1)|\} \leqq 2 M$. If possible, assume that $\max \{|x(0)|$, $|x(1)|\}>2 M$. If $|x(t)| \geqq M$ almost everywhere in $[0,1]$, then $\int_{0}^{1}|x(t)| d t \geqq M$ 
which with (5) gives $T(x)=0$. So $|x(t \pm)|=|x(0)|=|x(1)|>2 M$. This contradicts (5). Hence there is a subset $E$ of $[0,1]$ of positive measure such that $|x(t \pm)|<M$ for all $t \in E$. Let $t$ be any point of $E \cap S_{x}$. Then

$$
|x(0)-x(t)|+|x(t)-x(1)|>M .
$$

So, $T(x)>M$ which contradicts (5). Therefore $\max \{|x(0)|,|x(1)|\} \leqq 2 M$. Combining this with (6) we get $|x(t \pm)| \leqq 3 M$ for all $t \in(0,1)$.

Let $\left\{x_{n}(t)\right\}$ be any sequence of points in $Y$. Then

$$
\left|x_{n}(t \pm)\right| \leqq 3 M, 0<t<1 ;\left|x_{n}(0)\right|,\left|x_{n}(1)\right|<3 M
$$

and $V_{S}\left(x_{n} ;[0,1]\right)<3 M$. By theorem 3.4 , there is a subsequence $\left\{x_{n_{i}}(t)\right\}$ which converges to a function $x(t)$ in $X$ almost everywhere in $[0,1]$. We may choose $\left\{x_{n_{i}}(t)\right\}$ and take the function $x(t)$ such that $\left\{x_{n_{i}}(t)\right\}$ converges to $x(t)$ also at $t=0,1$. Let $\tau=\lim _{i \rightarrow \infty} \inf T\left(x_{n_{i}}\right)$. We choose a subsequence $\left\{T\left(x_{m_{i}}\right)\right\}$ of $\left\{T\left(x_{n_{i}}\right)\right\}$ which converges to $\tau$. By theorem 3.2, $\tau \geqq T(x)$. Let $K=\tau-T(x)$. We define the function $y(t)$ on $[0,1]$ as follows:

$$
\begin{aligned}
y(t) & =x(t), 0<t \leqq 1 \\
& =x(0)+K \text { for } t=0 \text { if } x(0)>x(0+), \\
& =x(0)-K \text { for if } x(0) \leqq x(0+) .
\end{aligned}
$$

It is clear that $y \in X$ and $T(y)=T(x)+K$. Further

$$
d\left(x_{m_{i}}, y\right)=\int_{0}^{1}\left|x_{m_{i}}(t)-x(t)\right| d t+\left|T\left(x_{m_{i}}\right)-\tau\right| .
$$

Then $d\left(x_{m_{i}}, y\right) \rightarrow 0$ as $i \rightarrow \infty$. We have

$$
d\left(y, x_{0}\right) \leqq d\left(x_{m_{i}}, y\right)+d\left(x_{m_{i}}, x_{0}\right) \leqq r+d\left(x_{m_{i}}, y\right) .
$$

Letting $i \rightarrow \infty$ we get $d\left(y, x_{0}\right) \leqq r$. Thus every sequence in $Y$ has a cluster point in $Y$. So by lemma ([12], Ch. $5, \S 4) Y$ is compact.

\section{The space $(\bar{X}, \rho)$}

Let $\bar{X}$ denote the family of all sets

$$
\{x\}^{-}=\{y ; y \in X \text { and } d(x, y)=0\}
$$

for $x \in X$. For convenience, we write $\bar{x}$ for $\{x\}^{-}$. For any two members $\bar{x}, \bar{y}$ of $\bar{X}$, let

$$
\rho(\bar{x}, \bar{y})=\inf \{d(\alpha, \beta) ; \alpha \in \bar{x} \text { and } \beta \in \bar{y}\} .
$$

Then $(\bar{X}, \rho)$ is a metric space ([12], Ch. $4, \S 15)$.

THEOREM 5.1. The space $(\bar{X}, \rho)$ is complete. 
Proof. Let $\left\{\bar{x}_{n}\right\}$ be any Cauchy sequence in $\bar{X}$. Then there is a positive number $M$ such that $\rho\left(\bar{x}_{n}, \bar{\theta}\right) \leqq M$ for all $n$, where $\theta(t)=0$ in [0,1]. Let $\alpha_{n}$ be any member of $\bar{x}_{n}$. Then $d\left(\alpha_{n}, \theta\right)=\rho\left(\bar{x}_{n}, \bar{\theta}\right) \leqq M$ for all $n$. Following the method of theorem 4.2 , we obtain a subsequence $\left\{\alpha_{n_{i}}(t)\right\}$ which converges to a function $x(t)$ in $X$ almost everywhere in $[0,1]$ such that $d\left(\alpha_{n_{i}}, x\right) \rightarrow 0$ as $i \rightarrow \infty$. Since $d\left(\alpha_{n_{i}}, x\right)=$ $\rho\left(\bar{x}_{n_{i}}, \bar{x}\right)$ it follows that the sequence $\left\{\bar{x}_{n_{i}}\right\}$ converges to $\bar{x}$ which implies that the sequence $\left\{\bar{x}_{n}\right\}$ converges to $\bar{x}$. This completes the proof.

Finally, the author is thankful to Dr. B. K. Lahiri for his kind help and suggestions in the preparation of the paper. Also, the author is thankful to the referee for his helpful suggestions.

\section{References}

[1] C. R. Adams, 'The space of functions of bounded variation and certain general spaces', Trans. Amer. Math. Soc., 40 (1936), $421-438$.

[2] C. R. Adams and J. A. Clarkson, 'On convergence in Variation', Bull. Amer. Math. Soc., 40 (1934), $413-417$.

[3] C. R. Adams and H. Lewy, 'On convergence in length', Duke Math. Journal 1 (1935), 19-26.

[4] C. R. Adams and A. P. Morse, 'On the space (BV)', Trans. Amer. Math. Soc. 42 (1937), $194-205$.

[5] L. Cesari, 'Sulle funzioni a Variazione limitata', Annali $R$. Scoula Normale Sup. Pisa, Series II, 5 (1936).

[6] L. Cesari, 'Funzioni continue a variazine limitata in Un insieme', Mem. Accad. Sci. 1 st. Bologna, cl. Sci. Fis (10) 2 (1946), 127-145.

[7] L. Cesari, 'Invertibilita in piccolo delle funzioni continue e teorema di derivazione', $\mathbf{M e m}$. Accad. Sci. 1st. Bologna cl. Sci. Fis (10) 3 (1947), 99-112.

[8] L. Cesari, 'Sul teorema di derivazione dulle funzioni variazione limitata in Un insieme', Mem. Accad. Sci. 1st. Bologna cl. Sci. Fis (10) 8 (1950-51), 223-229.

[9] L. Cesari, 'Variation, multiplicity and semi-continuity', Amer. Math. Monthly 65 (1958), $317-332$.

[10] R. L. Jeffery, 'Generalised integrals with respect to functions of bounded variation', Can. J. Math. 10 (1958), 617-628.

[11] K. Krickeberg, 'Distributionen functionen beschrankter variation und Lebesguescher inhalt nichtparamätrischer flachen', Annali Matem. Pura appl. (4) 44 (1957), 105-133.

[12] J. H. Kelley, General Topology (Van Nostrand, New York, 1955).

[13] I. P. Natanson, Theory of functions of a real variable. Vol. I, (Unger, New York, 1960).

[14] S. Saks, Theory of the integral (Warsaw, 1937; Dovev, New York, 1964).

Department of Mathematics

Jadavpur University

Calcutta-32, India 